\title{
Cylindrical Tightly Coupled Dipole Array Antenna
}

\author{
Hakjune Lee ${ }^{1} \cdot$ Minyoung Yoon ${ }^{2} \cdot$ Sangwook Nam ${ }^{2, *}$
}

\begin{abstract}
A cylindrical tightly coupled dipole array antenna (C-TCDA) based on Munk's planar TCDA theory, the elements of which are placed on the surface of a cylinder, is introduced and analyzed in this study. The explicit field components of the transverse electromagnetic (TEM) waves existing in the C-TCDA for both polarizations are given. Moreover, the embedding impedance characteristics of the CTCDA are presented and compared with those of planar TCDA. Analysis results show that the C-TCDA has similar impedance characteristics to the planar TCDA and thus can be implemented in a similar manner. The design of a dual-polarized omnidirectional octagonal TCDA, which has a 3.08:1 bandwidth (from $0.73 \mathrm{GHz}$ to $2.25 \mathrm{GHz}$ ) with a voltage standing wave radiation (VSWR) of less than 2 and a low gain variation of less than $1.8 \mathrm{~dB}$ at broadside for both polarizations, is also proposed. When scanning up to $\pm 30^{\circ}$ in the $\theta$ direction, the array operates in the same frequency band with a VSWR of less than 2.05 and a low gain variation of less than $2 \mathrm{~dB}$ for both polarizations.
\end{abstract}

Key Words: Array Antenna, Omnidirectional Antenna, Phased Array.

\section{INTRODUCTION}

In recent years, omnidirectional array antennas with the following characteristics have become necessary for wireless communication systems: (1) wide bandwidth to cover various frequency bands, (2) dual polarization for durable communication in complex environments, and (3) low gain variation in the horizontal plane for omnidirectional coverage [1,2]. Various omnidirectional antennas satisfying the above characteristics have been reported. Most of these antennas have dual polarizations and low gain variations in the horizontal plane. However, their bandwidths are quite limited, reaching only $25 \%$ [3] or $30 \%$ [4]. Antennas with an extended bandwidth in the vertical polarization of over $110 \%$ have been proposed [5-7], but their bandwidths in the horizontal polarization are still limited to below $65 \%$.
The tightly coupled dipole array (TCDA) proposed by Munk [8] can achieve ultra-wideband because of the compensation between the coupling capacitance of the neighboring dipole antennas and the inductance of the ground plane at low frequencies. Various types of dual-polarized TCDAs with very broad bandwidths have been introduced, for example, 5:1 [9], 3:1 [10], 7.35:1 [11], and 6:1 [12]. In developing various TCDA radiation patterns, a one-dimensional TCDA with a fan-shaped beam has also been presented $[13,14]$. However, studies on TCDA antennas have been limited to planar arrays for achieving pencil or fan beams.

In this study, we propose a cylindrical TCDA (C-TCDA) design for an omnidirectional radiation pattern, which has a wide bandwidth and dual polarization compared with conventional omnidirectional antennas. In Section II, we analyze the proposed C-TCDA and verify that it has similar impedance characteristics to planar TCDAs. The dual-polarized

Manuscript received September 15, 2018 ; Revised December 11, 2018 ; Accepted January 29, 2019. (ID No. 20180915-065J)

${ }^{1}$ The Electronics and Telecommunications Research Institute (ETRI), Daejeon, Korea.

${ }^{2}$ School of Electrical Engineering and Computer Science, Institute of New Media and Communications, Seoul National University, Seoul, Korea.

"Corresponding Author: Sangwook Nam (e-mail: snam@snu.ac.kr)

This is an Open-Access article distributed under the terms of the Creative Commons Attribution Non-Commercial License (http://creativecommons.org/licenses/by-nc/4.0) which permits unrestricted non-commercial use, distribution, and reproduction in any medium, provided the original work is properly cited.

(c) Copyright The Korean Institute of Electromagnetic Engineering and Science. All Rights Reserved. 
omnidirectional TCDA antenna design and its performances are introduced in Section III. The conclusions are presented in Section IV.

\section{THEORY OF THE C-TCDA}

In this section, the equations for the C-TCDA and its equivalent circuit are presented. We approach the C-TCDA analysis in a similar manner as the analysis of a planar TCDA. The cylindrical coordinates $(\rho, \varphi, z)$ are used, with the cylinder being parallel to the $z$-axis and the direction of wave propagation being along the $\rho$-axis. Two types of $\mathrm{C}$ TCDA with elements arranged in the $\varphi$-direction (horizontal polarization) and the $z$-direction (vertical polarization) are analytically solved. The cylindrical and planar TCDAs are then compared.

\section{Horizontally Polarized $C-T C D A$}

A schematic of the horizontally polarized C-TCDA is shown in Fig. 1(a). The horizontally polarized $N$ elements of the TCDA are arranged in the $\varphi$-direction with a period of $\varphi_{0}=2 \pi / N$ and repeated in the $z$-direction with a period of $b$. According to Wheeler's current sheet array theory, a twodimensionally periodic dipole array located in a plane can be equated to a unit dipole in a hypothetical waveguide that has a perfect electric conductor (PEC) and a perfect magnetic conductor (PMC) boundary conditions [15]. Similar to a planar array in Fig. 1(a), the cylindrically infinite dipole array is equivalent to the unit dipole inside the hypothetical waveguide, as shown in Fig. 1(b). We assume that the medium inside the waveguide is filled with an isotropic, homogeneous, and lossless dielectric material with a permittivity $\varepsilon$ and permeability $\mu_{0}$. The boundaries parallel to the $z$-axis are PEC, and the other boundaries orthogonal to the $z$-axis are PMC. Therefore, the boundary conditions are written as

$$
\begin{gathered}
E_{\rho}(\rho, \phi, z)=E_{z}(\rho, \phi, z)=0 \text { at } \phi=0, \phi_{0}, \\
H_{\rho}(\rho, \phi, z)=H_{\phi}(\rho, \phi, z)=0 \text { at } z=0, b .
\end{gathered}
$$

The field components inside the waveguide can be obtained by solving the Helmholtz wave equation using the separation-of-variables technique [15]. The current of the dipole flows in the $\varphi$-direction, and thus the magnetic wave modes transverse to the $\varphi$-direction exist. For the magnetic modes transverse to $\varphi$ that propagate only in the $+\rho$ direction (radiation direction), the vector potential satisfying the boundary conditions in Eqs. (1) and (2) can be written as

$$
\mathbf{A}_{p m}=\psi_{p m} \hat{\phi}=B_{p m} H_{\frac{p \pi}{\phi_{0}}}^{(2)}\left(k_{\rho} \rho\right) \cos \left(\frac{p \pi}{\phi_{0}} \phi\right) \cos \left(\frac{m \pi}{b} z\right) \hat{\phi},
$$

with

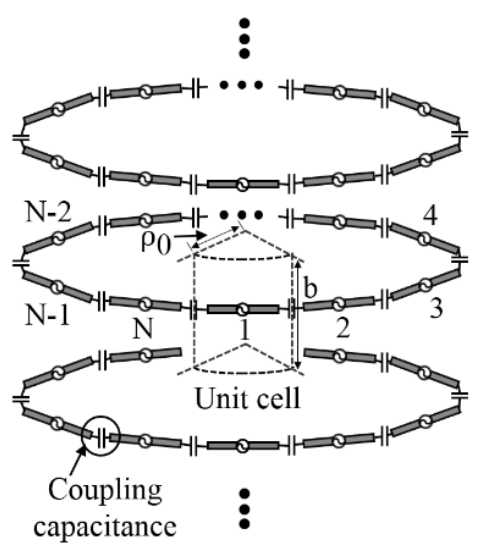

(a)

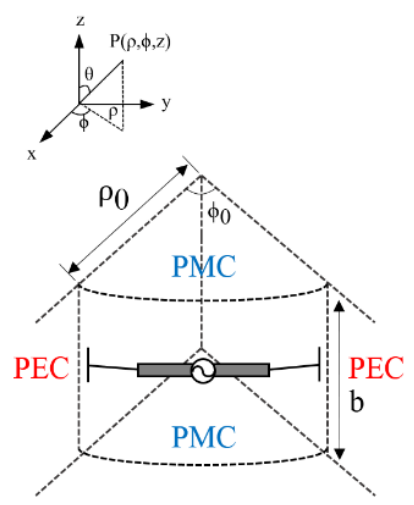

(b)
Fig. 1. (a) Horizontally polarized C-TCDA. (b) Unit cell of the horizontally polarized C-TCDA (without a ground plane and a superstrate).

$$
k_{\rho}=\sqrt{k^{2}-\left(\frac{m \pi}{b}\right)^{2}},
$$

where $p=0,1,2, \ldots$ and $m=0,1,2, \ldots$ The variable $B_{p m}$ is the coefficient of the $p m$ mode. The wave number, $k$, is given by

$$
k=\omega \sqrt{\mu_{0} \varepsilon} .
$$

Therefore, the fundamental mode is $\mathrm{TM}_{00}$ transverse to $\varphi$, and the corresponding vector potential is

$$
\mathbf{A}_{00}=\psi_{00} \hat{\phi}=B_{00} H_{0}^{(2)}(k \rho) \hat{\phi} .
$$

The field components of the dominant mode are obtained by substituting Eq. (5) into the Maxwell equations. The results are as follows:

$$
\begin{gathered}
E_{\rho}(\rho, \phi, z)=0, \\
E_{\phi}(\rho, \phi, z)=B_{00} \frac{1}{j \omega \varepsilon} k^{2} H_{0}^{(2)}(k \rho), \\
E_{z}(\rho, \phi, z)=0, \\
H_{\rho}(\rho, \phi, z)=0, \\
H_{\phi}(\rho, \phi, z)=0, \\
H_{z}(\rho, \phi, z)=B_{00}\left(\frac{1}{\rho} H_{0}^{(2)}(k \rho)-k H_{1}^{(2)}(k \rho)\right) .
\end{gathered}
$$

Only $E_{\phi}$ and $H_{\mathrm{z}}$ exist, signifying that the mode is TEM to the $\rho$ mode. As $E_{\phi}$ and $H_{\mathrm{z}}$ are functions of $\rho$, the hypothetical waveguide can be regarded as a transmission line with characteristic impedance as a function of $\rho$. The characteristic impedance of the transmission line for the horizontal polarization is given by 


$$
Z_{c h}(\rho)=\frac{\rho \phi_{0}}{b} \frac{E_{\phi}}{H_{z}}=\frac{\rho \phi_{0}}{b} \frac{\frac{1}{j \omega \varepsilon} k^{2} H_{0}^{(2)}(k \rho)}{\frac{1}{\rho} H_{0}^{(2)}(k \rho)-k H_{1}^{(2)}(k \rho)} .
$$

The phase constant for the $\mathrm{TM}_{00}$ mode is given by [12]

$$
\beta(\rho)=\frac{2}{\pi \rho} \frac{1}{J_{0}^{2}(k \rho)+N_{0}^{2}(k \rho)} .
$$

The cutoff frequencies of the $p m$ mode is given by

$$
f_{c}=\frac{m}{2 \pi b \sqrt{\mu_{0} \varepsilon}}=\frac{m c}{2 \pi b} .
$$

The grating lobe occurs over the first cutoff frequency $(m$ $=1$ ) when scanning in the $\theta$-direction because $b \geq \lambda / 2$. All modes where $p>0$ and $m=0$ are possible, but we focus on only the dominant mode for simplicity.

The equivalent circuit of the C-TCDA unit cell with a ground plane and a superstrate is shown in Fig. 2. Let the distances between the center of the cylinder and the ground plane, the dipole antenna, and the top of the superstrate be $\rho_{\mathrm{g}}, \rho_{\text {ant }}$, and $\rho_{\text {sup }}$, respectively. The variables $Z_{0 \mathrm{~h}}$ and $Z_{\text {suph }}$ are the characteristic impedance of free space and the superstrate for the horizontal polarization, respectively, and they are obtained by substituting the vacuum permittivity, $\varepsilon_{0}$, and the superstrate permittivity, $\varepsilon_{\text {sup }}$, for $\varepsilon$ in Eq. (13). The variables $\beta_{0}$ and $\beta_{\text {sup }}$ are the phase constant of free space and the superstrate, respectively, which are obtained by substituting $\varepsilon_{0}$ and $\varepsilon_{\text {sup }}$ for $\varepsilon$ in Eq. (14). The embedding impedances $Z_{\mathrm{u}}$ and $Z_{\mathrm{d}}$ are the impedances toward the outward direction and inward direction in the dipole antenna, respectively. $Z_{\text {ant }}$ is the antenna impedance, which is composed of the dipole inductance, $L_{\text {dipole }}$, and the coupling capacitance, $C_{\text {coupling. }}$ Therefore, the input impedance is given by $Z_{\text {in }}=Z_{\text {ant }}+Z_{\mathrm{u}} / / Z_{\mathrm{d}}=j \omega$ $L_{\text {dipole }}+1 / j \omega C_{\text {coupling }}+Z_{\mathrm{u}} / / Z_{\mathrm{d}}$.

\section{Vertically Polarized $C-T C D A$}

A schematic of the vertically polarized C-TCDA is shown in Fig. 3(a). The vertically polarized $N$ elements of the

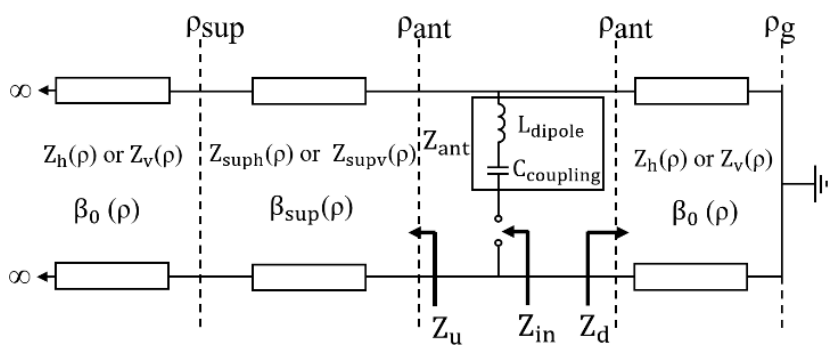

Fig. 2. Equivalent circuit of the C-TCDA. The subscripts $h$ and suph correspond to horizontal polarization, and the superscript $v$ and supv correspond to vertical polarization.

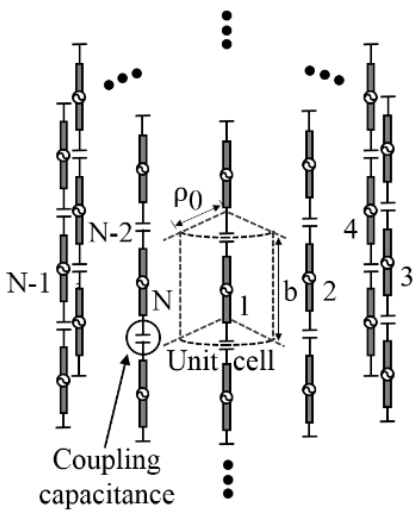

(a)

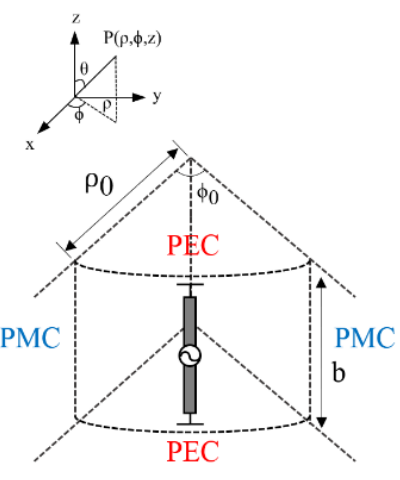

(b)
Fig. 3. (a) Vertically polarized C-TCDA. (b) Unit cell of the vertically polarized C-TCDA (without a ground plane and a superstrate).

TCDA are arranged in the $\varphi$-direction with a period of $\varphi_{0}=$ $2 \pi / N$ and repeated in the $z$-direction with a period of $b$. Similar to the horizontally polarized C-TCDA, the arrays are equivalent to a unit dipole existing in the hypothetical waveguide with PEC and PMC boundary conditions, as shown in Fig. 3(b). We also assume that the medium inside the waveguide is filled with an isotropic, homogeneous, and lossless dielectric material, with permittivity $\varepsilon$ and permeability $\mu_{0}$. Contrary to the horizontal polarization case, the boundaries parallel to the $z$-axis are PMC, and the other boundaries orthogonal to the $z$-axis are PEC. Thus, the boundary conditions are written as

$$
\begin{aligned}
& E_{\rho}(\rho, \phi, z)=E_{\phi}(\rho, \phi, z)=0 \text { at } z=0, b, \\
& H_{\rho}(\rho, \phi, z)=H_{z}(\rho, \phi, z)=0 \text { at } \phi=0, \phi_{0} .
\end{aligned}
$$

The field components inside the waveguide can be obtained in a similar way to that in the horizontal polarization. The current of the dipole flows in the $z$-direction, and therefore the modes transverse to the $z$-axis exist. For the modes transverse to the $z$-direction that propagate only in the $+\rho$ direction (radiation direction), the vector potential satisfying the boundary conditions in Eqs. (16) and (17) can be written as

$$
\mathbf{A}_{p m}=\psi_{p m} \hat{z}=C_{p m} \cos \left(\frac{p \pi}{\phi_{0}} \phi\right) \cos \left(\frac{m \pi}{b} z\right) H_{\frac{p \pi}{\phi_{0}}}^{(2)}\left(k_{\rho} \rho\right) \hat{z},
$$

with $k_{\rho}$ given in Eq. (4), where $p=0,1,2, \ldots$, and $m=0,1$, $2, \ldots . C_{p m}$ is the coefficient of the $p m$ mode. The wave number, $k$, is given in Eq. (5). Thus, the fundamental mode is $\mathrm{TM}_{00}$ transverse to $z$, and the corresponding vector potential is

$$
\mathbf{A}_{00}=\psi_{00} \hat{z}=C_{00} H_{0}^{(2)}(k \rho) \hat{z} .
$$

The field components of the dominant mode are obtained from substituting Eq. (19) into the Maxwell equations. The 
results are as follows:

$$
\begin{gathered}
E_{\rho}(\rho, \phi, z)=0, \\
E_{\phi}(\rho, \phi, z)=0, \\
E_{z}(\rho, \phi, z)=C_{00} \frac{1}{j \omega \varepsilon} k^{2} H_{0}^{(2)}(k \rho), \\
H_{\rho}(\rho, \phi, z)=0, \\
H_{\phi}(\rho, \phi, z)=C_{00} k H_{1}^{(2)}(k \rho), \\
H_{z}(\rho, \phi, z)=0 .
\end{gathered}
$$

Only $E_{z}$ and $H_{\phi}$ exist, signifying that the mode is TEM transverse to $\rho$. Furthermore, because $E_{\mathrm{z}}$ and $H_{\phi}$ are functions of $\rho$, the hypothetical waveguide can be regarded as a transmission line, the characteristic impedance of which is a function of $\rho$. The characteristic impedance of the transmission line for the vertical polarization is

$$
Z_{c v}(\rho)=-\frac{b}{\rho \phi_{0}} \frac{E_{z}}{H_{\phi}}=-\frac{b}{\rho \phi_{0}} \frac{\frac{1}{j \omega \varepsilon} k H_{0}^{(2)}(k \rho)}{H_{1}^{(2)}(k \rho)} .
$$

The phase constant for the $\mathrm{TM}_{00}$ mode and the cutoff frequencies of the $p m$ mode are the same as those for the horizontal polarization given in Eqs. (14) and (15), respectively. The grating lobe occurs at the first cutoff frequency $(m=1)$ when scanning in the $\theta$-direction, as $b \geq \lambda / 2$. All modes where $p>0$ and $m=0$ are possible, but we consider only the dominant mode for simplicity.

The equivalent circuit of the TCDA unit cell with a ground plane and a superstrate is shown in Fig. 2. $Z_{0 \mathrm{v}}$ and $Z_{\text {supv }}$ are the characteristic impedance of free space and the superstrate for the vertical polarization, respectively, and they are obtained by substituting $\varepsilon_{0}$ and $\varepsilon_{\text {sup }}$ for $\varepsilon$ in Eq. (26). The variables $\beta_{0}, \beta_{\text {sup }}, Z_{\mathrm{u}}, Z_{\mathrm{d}}, Z_{\text {ant }}$, and $Z_{\text {in }}$ are identical to those of the horizontally polarized C-TCDA.

\section{Comparison with the Planar TCDA}

The equivalent circuit of the planar TCDA proposed in [10] is shown in Fig. 4. Let $a$ and $d$ be the lengths of the hypothetical waveguide of the planar TCDA unit cell that are parallel and orthogonal to the dipole direction, respectively. The characteristic impedance of the waveguide as given by [11] is

$$
Z_{c p}(\rho)=\frac{a}{d} \sqrt{\frac{\mu}{\varepsilon}},
$$

and the wave number $k$ is given in Eq. (5). The distance between the ground plane and the antenna is $h_{\text {ant }}$ and that between the antenna and the superstrate is $h_{\text {sup }} . Z_{\text {op }}$ and $Z_{\text {supp }}$

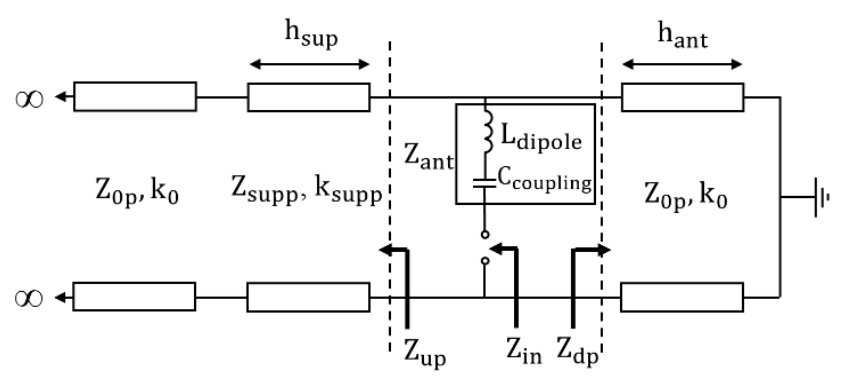

Fig. 4. Equivalent circuit of the planar TCDA.

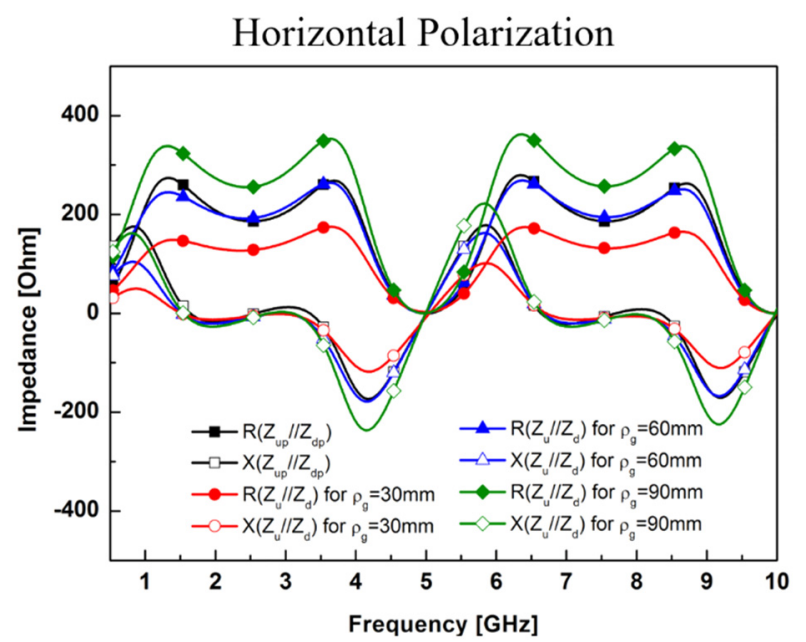

(a)

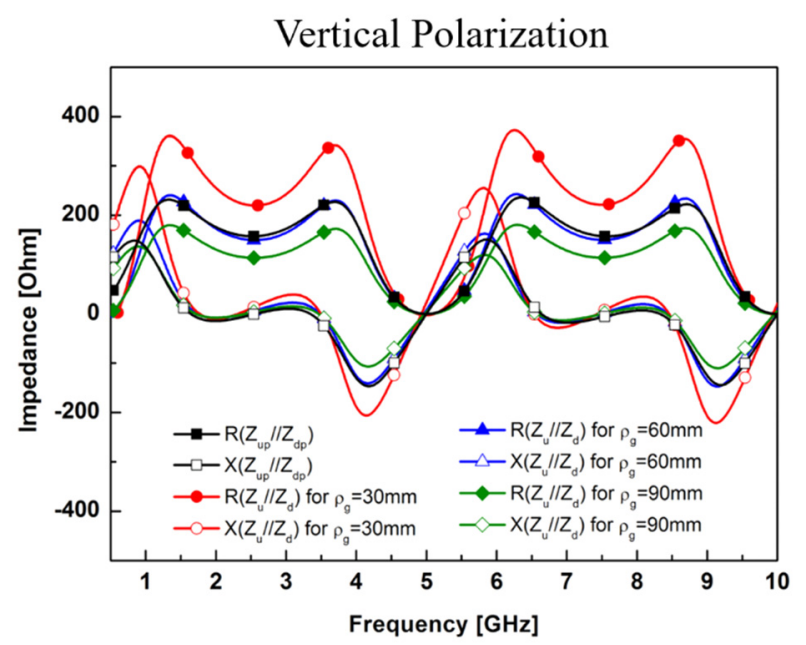

(b)

Fig. 5. Resistance and reactance of $Z_{\mathrm{u}} / / Z_{\mathrm{d}}$ and $Z_{\mathrm{up}} / / Z_{\mathrm{dp}}$ for horizontal polarization (a) and vertical polarization (b).

are obtained by substituting $\varepsilon_{0}$ and $\varepsilon_{\text {sup }}$, respectively, for $\varepsilon$ in Eq. (17). The variables $k_{0}$ and $k_{\text {supp }}$ are obtained by substituting $\varepsilon_{0}$ and $\varepsilon_{\text {sup }}$, respectively, for $\varepsilon$ in Eq. (5).

The planar TCDA can have a wide bandwidth because the input impedance, $Z_{\text {in }}=Z_{\text {ant }}+Z_{\text {up }} / / Z_{\mathrm{dp}}$, is matched for a wide range of frequencies. $Z_{\text {ant }}$ is determined by the dipole antenna shape and the gap size between the neighboring dipoles. However, the embedding impedance, $Z_{\mathrm{u}} / / Z_{\mathrm{d}}$ or $Z_{\mathrm{up}} / /$ 
$Z_{\mathrm{dp}}$, is inherently determined by the shape of the array structure.

A comparison between $Z_{\mathrm{u}} / / Z_{\mathrm{d}}$ and $Z_{\mathrm{up}} / / Z_{\mathrm{dp}}$ for both polarizations is presented in Fig. 5. For the C-TCDA, the relative distances $\rho_{\text {ant }}-\rho_{\mathrm{g}}$ and $\rho_{\text {sup }}-\rho_{\text {ant }}$ are fixed to $30 \mathrm{~mm}$ and $20 \mathrm{~mm}$, respectively. Then, $Z_{\mathrm{u}} / / Z_{\mathrm{d}}$ is plotted for $\rho_{\mathrm{g}}=30,60$, and $90 \mathrm{~mm}$. The other specific values are $\varepsilon_{\text {sup }}=2.2 \times \varepsilon_{0}, \varphi_{0}$ $=\pi / 4(N=8)$, and $b=65 \mathrm{~mm}$. For the planar TCDA, $h_{\mathrm{ant}}=$ $\rho_{\text {ant }}-\rho_{\mathrm{g}}=30 \mathrm{~mm}, h_{\text {sup }}=\rho_{\text {sup }}-\rho_{\text {ant }}=20 \mathrm{~mm}$, and $\varepsilon_{\text {sup }}=2.2$ $\times \varepsilon_{0}$ are commonly used. The lengths of the hypothetical waveguide are chosen as $a=60 \mathrm{~mm} \times \varphi_{0} \approx 70.7 \mathrm{~mm}$ and $d$ $=65 \mathrm{~mm}$ for the horizontal polarization and $a=65 \mathrm{~mm}$ and $d=60 \mathrm{~mm} \times \varphi_{0} \approx 70.7 \mathrm{~mm}$ for the vertical polarization. As a result, $Z_{\mathrm{u}} / / Z_{\mathrm{d}}$ when $\rho_{\mathrm{g}}=60 \mathrm{~mm}$ and $Z_{\mathrm{up}} / / Z_{\mathrm{dp}}$ have similar values for both polarizations. Furthermore, when $\rho_{\mathrm{g}}$ increases, the amplitude of $Z_{\mathrm{u}} / / Z_{\mathrm{d}}$ increases for the horizontal polarization and decreases for the vertical polarization. As the $\mathrm{C}$ TCDA has similar impedance characteristics to the planar TCDA, the C-TCDA can achieve a wide bandwidth as the planar TCDA by adjusting the antenna impedance.

\section{DESIGN OF DUAL-POLARIZED C-TCDA}

In the previous section, we verified that the C-TCDA antennas have similar impedance characteristics to the planar TCDA antenna. In this section, a dual-polarized C-TCDA antenna design and its performances are presented. For simplicity of the design, the cylinder is approximated as an octagon.

\section{Design}

The proposed dual-polarized C-TCDA is presented in Fig. 6. Fig. 6(a) shows a layer of the array antenna, which consists of 8 unit cells $(N=8)$ and has a periodic boundary in

the $z$-direction. A unit cell of the C-TCDA antenna is illustrated in Fig. 6(b). Printed circuit boards (PCBs), which are the horizontal and vertical polarization antennas, are orthogonally soldered to the ground plane. The vertical polarization dipole antenna is located away from the center of the unit cell ground plane for low coupling between both polarizations. The substrate of both polarization antennas is designed using the Rogers RT/Duroid 5880 PCB with a substrate thickness of $0.508 \mathrm{~mm}$, dielectric constant of 2.2, and dielectric loss tangent of 0.001 . The superstrate is implemented by polytetrafluoroethylene (PTFE) with a dielectric constant of 2.1 and a dielectric loss tangent of 0.0005 . The superstrate is used for impedance matching in a low-profile array antenna [8]. The front and back sides of the horizontal polarization dipole are presented in Fig. 6(c) and (d), and the front and back sides of the vertical polarization dipole are presented in Figs. 6(e) and (f), respectively. The feeding line of the front side of both dipoles is connected to an subminiature version A (SMA) connector and that of the back side of both dipoles is connected with the ground plane (unbalanced feeding). Both dipoles, except the feeding line, are rotationally symmetric and have shorting posts connected with the ground plane to adjust the common mode resonance frequency analyzed in [9]. The specific values are as follows: $\rho_{\mathrm{g}}$ $=60 \mathrm{~mm}, b=30 \mathrm{~mm}, h_{\mathrm{s}}=20 \mathrm{~mm}, w_{\text {sup }}=70 \mathrm{~mm}, b=65$ $\mathrm{mm}, l_{\mathrm{g}}=50 \mathrm{~mm}, v=15 \mathrm{~mm}, l_{\mathrm{h}}=58.7 \mathrm{~mm}, g_{\mathrm{h}}=0.15 \mathrm{~mm}$, $l_{\mathrm{a} 1}=12.14 \mathrm{~mm}, l_{\mathrm{a} 2}=2 \mathrm{~mm}, w_{\mathrm{h} 1}=1 \mathrm{~mm}, w_{\mathrm{h} 2}=w_{\mathrm{h} 3}=1.5$ $\mathrm{mm}, h_{\mathrm{h}}=30 \mathrm{~mm}, s_{\mathrm{h}}=7.7 \mathrm{~mm}, \theta_{\mathrm{h}}=35^{\circ}, \theta_{\mathrm{c}}=22.5^{\circ}, l_{\mathrm{v}}=63$ $\mathrm{mm}, w_{\mathrm{v} 1}=w_{\mathrm{v} 2}=w_{\mathrm{v} 3}=1 \mathrm{~mm}, h_{\mathrm{v}}=30 \mathrm{~mm}, s_{\mathrm{v}}=12.7 \mathrm{~mm}, \theta_{\mathrm{v}}$ $=45^{\circ}, s=0.1 \mathrm{~mm}, h_{\mathrm{b}}=45 \mathrm{~mm}, h_{1}=26 \mathrm{~mm}$, and $h_{2}=4 \mathrm{~mm}$. The C-TCDA was simulated by CST Microwave Studio.

\section{Performance}

Fig. 7 shows the simulated voltage standing wave ratio

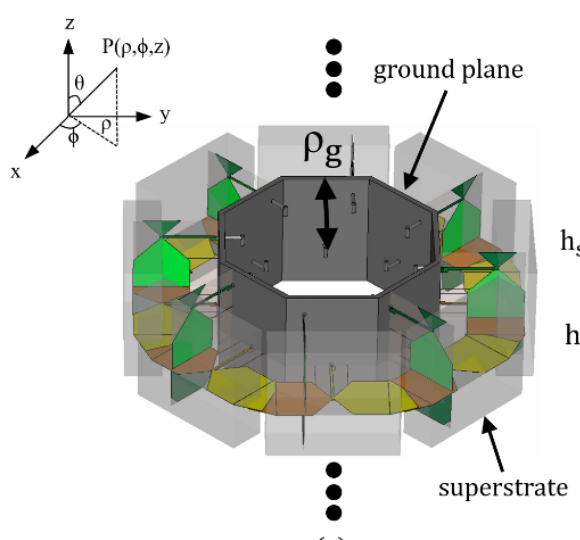

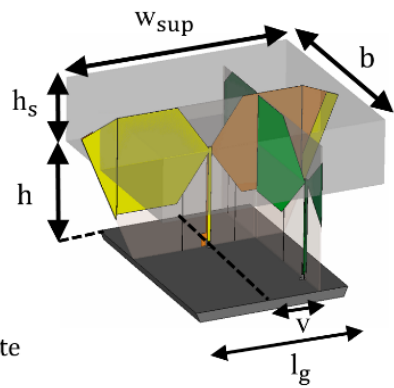

(b)

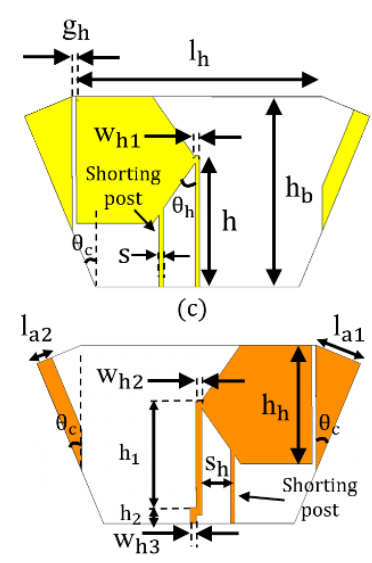

(d)

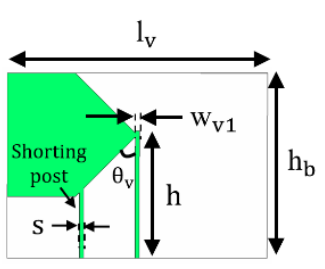

(e)

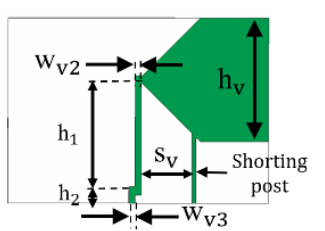

(f)

Fig. 6. Configuration of the proposed octagonal, dual-polarized TCDA antenna (not scaled): (a) one layer, (b) unit cell, (c) front side of the horizontally polarized dipole, (d) back side of the horizontally polarized dipole, (e) dront side of the vertically polarized dipole, and (f) back side of the vertically polarized dipole. 
(VSWR) and total efficiency for the C-TCDA antenna. The overlapped bandwidth is $3.08: 1$ (from $0.73 \mathrm{GHz}$ to 2.25 $\mathrm{GHz}$ ) with VSWR less than 2. The coupling between the horizontal and vertical polarizations is under $18 \mathrm{~dB}$, and the total efficiency, including return loss and radiation efficiency, is over $86 \%$ for both polarizations in the frequency band.

A phase difference between neighboring layers is necessary to scan in the $\theta$-direction while maintaining the omnidirectional pattern. Let the phase difference $\Delta \theta$ be given by

$$
\Delta \theta=\frac{2 \pi f \mathrm{~b} \sin \theta_{S}}{\mathrm{c}},
$$

where $\theta_{\mathrm{s}}$ is the desired scan angle. Fig. 8 shows the VSWR versus the frequency graphs for $\Delta \theta=29.25^{\circ}, 50^{\circ}, 70^{\circ}$, and $87.75^{\circ}$. If $\left|\theta_{\mathrm{s}}\right| \leq 30^{\circ}, f \geq c \Delta \theta / \pi b$, as obtained from the modified Eq. (28). The minimum frequency corresponding to $\Delta \theta$ and the upper frequency, $2.25 \mathrm{GHz}$, are marked by

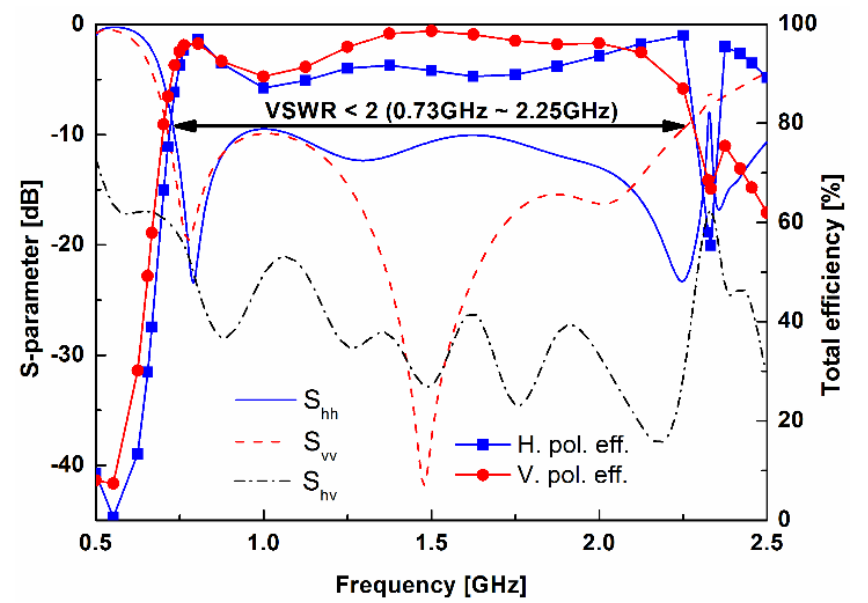

Fig. 7. $S$-parameter and total efficiency of the proposed array antenna.
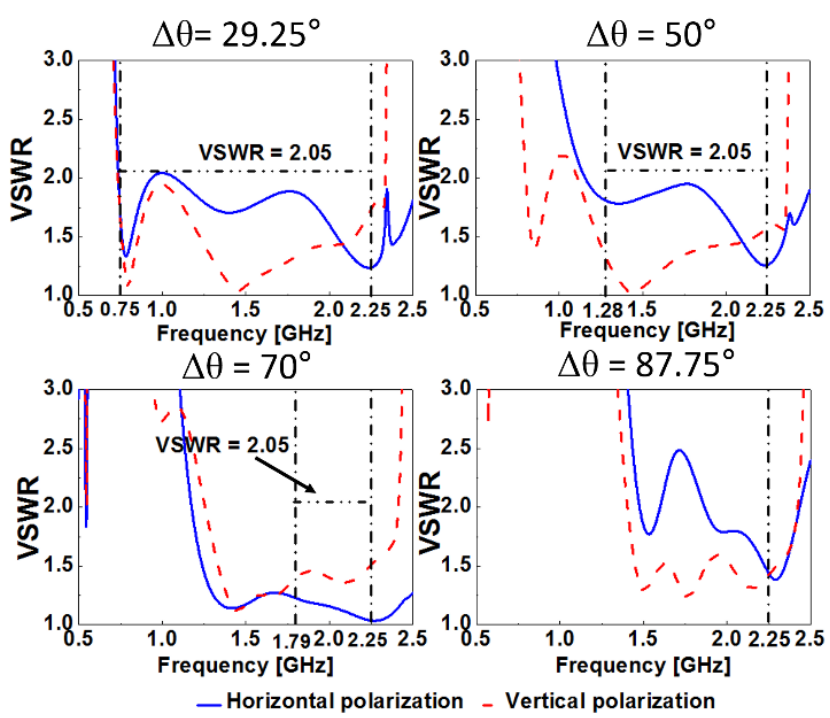

Fig. 8. VSWR versus frequency graphs when the $\Delta \theta$ is $29.25^{\circ}, 50^{\circ}$, $70^{\circ}$, or $87.75^{\circ}$. dash-dot lines in Fig. 8. The VSWRs are under 2.05 in both cases, and thus the octagonal C-TCDA can scan in the $\theta$ direction up to $\pm 30^{\circ}$ with a low VSWR.

The normalized radiation patterns of the eight-layer CTCDA for the horizontal and vertical polarizations are presented in Fig. 9. As expected, the $E_{\phi}$ components are predominantly observed when the horizontal polarization dipoles are excited, and the $E_{\theta}$ components are predominantly observed when the vertical polarization dipoles are excited. The C-TCDA has good omnidirectional patterns in the azimuthal plane for both polarizations. In the elevation plane pattern, the C-TCDA can scan in the desired direction up to $\pm 30^{\circ}$ for both polarizations. Most of the patterns show a low cross-polarization under $-10 \mathrm{~dB}$ compared with the copolarization and a sidelobe under $-10 \mathrm{~dB}$.

The realized gain and gain variation of the eight-layer $\mathrm{C}$ TCDA versus frequency graphs radiating at broadside $\left(0^{\circ}\right)$ and scanning to $30^{\circ}$ are shown in Fig. 10. The gain and gain variation values for the scan angles between $0^{\circ}$ and $30^{\circ}$ are between the values when the scan angle is $0^{\circ}$ and $30^{\circ}$. The realized gain has a range of $4-9.3 \mathrm{~dB}$ and 3.6-5.7 $\mathrm{dB}$ for $0^{\circ}$ and up to $\pm 30^{\circ}$, respectively. Moreover, the gain variation in the azimuthal plane is lower than $1.8 \mathrm{~dB}$ and $2 \mathrm{~dB}$ for $0^{\circ}$ and up to $\pm 30^{\circ}$, respectively.

For practical implementation, the C-TCDA elements must be finite in the $z$-direction. Most studies on planar TCDAs show that TCDAs using $8 \times 8$ or more elements perform similarly to the infinite case [10-12]. Therefore, there should be little difference in performance between a practical $\mathrm{N} \times \mathrm{M}(\geq 8) \mathrm{C}$-TCDA and the $\mathrm{N} \times$ infinite $\mathrm{C}$ TCDA simulated in this design.

\section{CONCLUSION}

We analyzed a C-TCDA and proposed the design of a wideband dual-polarized array antenna for an omnidirectional pattern. Similar to that in the planar TCDA, the radiation wave can exist as a TEM mode in the C-TCDA. Moreover, the embedding impedance characteristics of the C-TCDA for both polarizations resemble those of the planar TCDA. The proposed C-TCDA antenna has a wide bandwidth of 3.08:1 (0.73-2.25 GHz) with an omnidirectional radiation pattern in the horizontal plane, a low cross-polarization level of $10 \mathrm{~dB}$, and a wide scanning angle of up to $\pm 30^{\circ}$. We consider that our C-TCDA theory and design can be applied to modern communication systems, such as indoor distributed antenna systems, base station antennas, and conformal array antenna systems for electric warfare. 


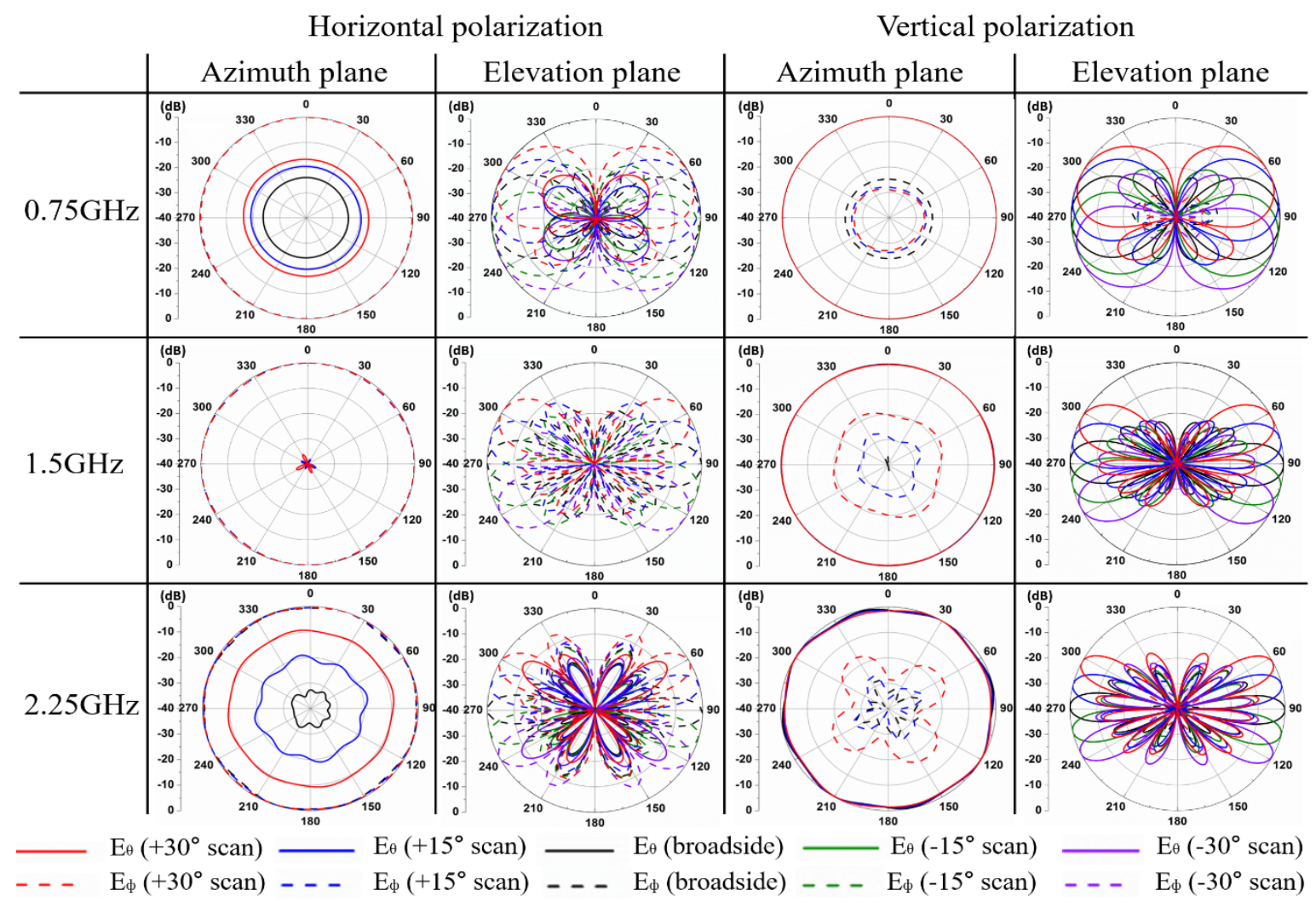

Fig. 9. Normalized radiation patterns for horizontal polarization and vertical polarization.

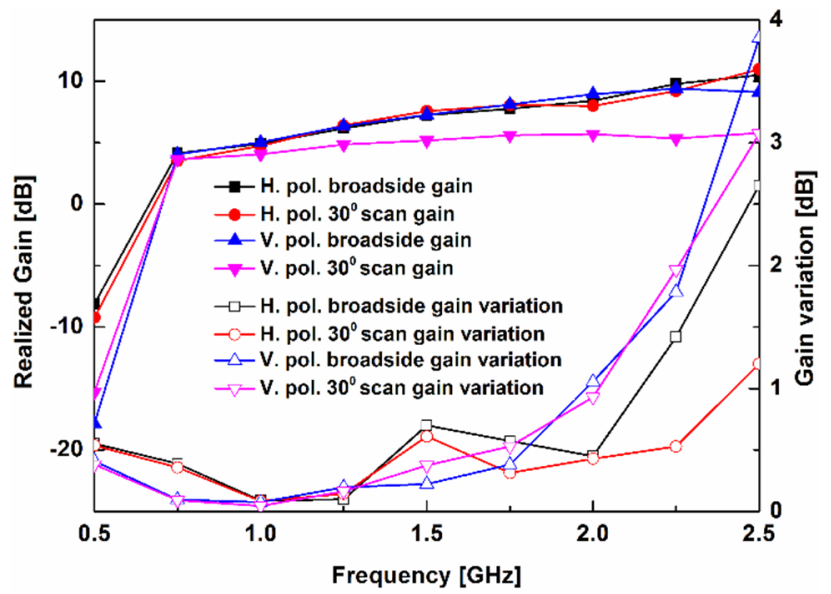

Fig. 10. Realized gain and gain variations in the azimuthal plane for both polarizations.

The authors gratefully acknowledge the support of the Electronic Warfare Research Center at Gwangju Institute of Science and Technology, originally funded by the Defense Acquisition Program Administration and the Agency for Defense Development. This work was supported by the Brain BK 21 Plus Project in 2018.

\section{REFERENCES}

[1] K. L. Wong, F. R. Hsiao, and T. W. Chiou, "Omnidi- rectional planar dipole array antenna," IEEE Transactions on Antennas and Propagation, vol. 52, no. 2, pp. 624-628, 2004.

[2] Y. X. Guo, K. W. Khoo, and L. C. Ong, "Wideband dual-polarized patch antenna with broadband baluns," IEEE Transactions on Antennas and Propagation, vol. 55, no. 1, pp. 78-83, 2007.

[3] X. Quan and R. Li, "A broadband dual-polarized omnidirectional antenna for base stations," IEEE Transactions on Antennas and Propagation, vol. 61, no. 2, pp. 943-947, 2013.

[4] Y. Fan, X. Liu, B. Liu, and R. Li, "A broadband dualpolarized omnidirectional antenna based on orthogonal dipoles," IEEE Antennas and Wireless Propagation Letters, vol. 15, pp. 1257-1260, 2016.

[5] X. W. Dai, Z. Y. Wang, C. H. Liang, X. Chen, and L. T. Wang, "Multiband and dual-polarized omnidirectional antenna for 2G/3G/LTE application," IEEE Antennas and Wireless Propagation Letters, vol. 12, pp. 1492-1495, 2013.

[6] F. Jolani, Y. Yu, and Z. Chen, "A novel broadband omnidirectional dual-polarized MIMO antenna for $4 \mathrm{G}$ LTE applications," in Proceedings of 2014 IEEE International Wireless Symposium (IWS), Xian, China, pp. 1-4.

[7] H. Huang, Y. Liu, and S. Gong, "Broadband dual-polarized omnidirectional antenna for 2G/3G/LTE/WiFi 
applications," IEEE Antennas and Wireless Propagation Letters, vol. 15, pp. 576-579, 2016.

[8] B. A. Munk, Finite Antenna Arrays and FSS. New York, NY: Wiley-IEEE Press, 2003.

[9] S. S. Holland and M. N. Vouvakis, "The planar ultrawideband modular antenna (PUMA) array," IEEE Transactions on Antennas and Propagation, vol. 60, no. 1, pp. 130-140, 2012.

[10] S. S. Holland, D. H. Schaubert, and M. N. Vouvakis, "A 7-21 GHz dual-polarized planar ultrawideband modular antenna (PUMA) array," IEEE Transactions on Antennas and Propagation, vol. 60, no. 10, pp. 45894600, 2012.

[11] J. P. Doane, K. Sertel, and J. L. Volakis, "A wideband, wide scanning tightly coupled dipole array with integrated balun (TCDA-IB)," IEEE Transactions on Antennas and Propagation, vol. 61, no. 9, pp. 4538-4548,

\section{Hakjune Lee}

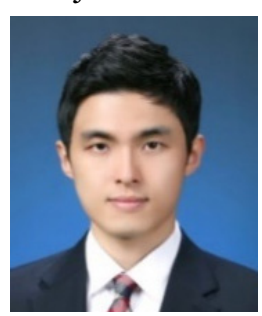

received his B.S. and M.S. degrees in electrical and computer engineering from Seoul National University, Seoul, Korea, in 2014 and 2016, respectively. Since 2016, he has been a researcher at the Electronics and Telecommunications Research Institute, Daejeon, Korea. His current research interests include ultrawideband array antennas and metamaterial.

Minyoung Yoon

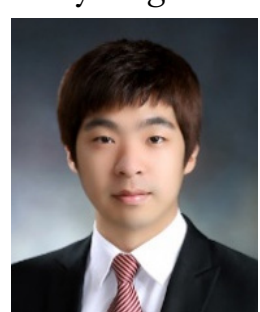

received his B.S. degree in electronic engineering from Ajou University, Suwon, Korea, in 2013. He is currently working toward his Ph.D. degree in electronic engineering in Seoul National University, Seoul, Korea. His main research interests include analog design based on a wideband conformal antenna system.
2013.

[12] M. Novak and J. L. Volakis, "Ultrawideband antennas for multiband satellite communications at UHF-Ku frequencies," IEEE Transactions on Antennas and Propagation, vol. 63, no. 4, pp. 1334-1341, 2015.

[13] H. Lee and S. Nam, "A dual-polarized 1-D tightly coupled dipole array antenna," IEEE Transactions on Antennas and Propagation, vol. 65, no. 9, pp. 4511-4518, 2017.

[14] Y. Wang, L. Zhu, H. Wang, Y. Luo, and G. Yang, "A compact, scanning tightly coupled dipole array with parasitic strips for next-generation wireless applications," IEEE Antennas and Wireless Propagation Letters, vol. 17, no. 4, pp. 534-537, 2018.

[15] R. F. Harrington, Time-Harmonic Electromagnetic Fields. New York, NY: Wiley-IEEE Press, 2001.

Sangwook Nam

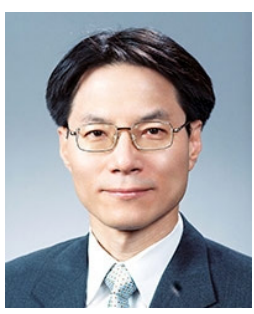

received his B.S. degree from Seoul National University, Seoul, Korea, in 1981, M.S. degree from the Korea Advanced Institute of Science and Technology, Seoul, Korea, in 1983, and Ph.D. degree from The University of Texas at Austin, Austin, TX, USA, in 1989 all in electrical engineering. From 1983 to 1986 , he was a researcher at the Gold Star Central Research Laboratory, Seoul, Korea. Since 1990, he has been a professor at the School of Electrical Engineering and Computer Science, Seoul National University. His research interests include analysis/design of electromagnetic structures, antennas, and microwave active/passive circuits. 